\title{
Le système cardio-vasculaire et sa régulation par le système nerveux autonome : modélisation et mesures
}

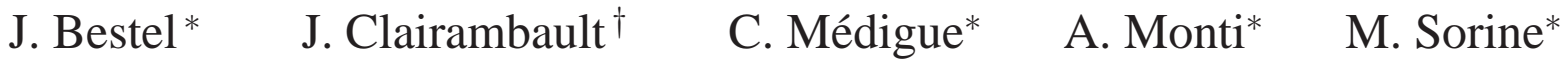 \\ Mai 1999
}

\begin{abstract}
Résumé
Le système cardio-vasculaire (SCV) est modélisé dans son ensemble, avec ses différents compartiments : pompe cardiaque, petite et grande circulation, circuit artériel et circuit veineux. Les problèmes de modélisation sont abordés sous l'angle de la commande, c'est-à-dire en fonction d'une mission à remplir pour le SCV et son contrôleur à court terme, le système nerveux autonome (SNA). Deux aspects du modèle sont développés ici : d'une part, la pompe cardiaque, intégrant un modèle non linéaire de muscle, et son insertion dans la circulation sanguine, d'autre part l'arc baroréflexe, principale boucle de régulation à court terme de la pression artérielle. Des applications cliniques en cours analysant le gain de cet arc baroréflexe sont également mentionnées.
\end{abstract}

\section{Le système cardio-vasculaire vu comme un système contrôlé}

(Résumé de [1], consultable en annexe ; pour plus de détails, on pourra se référer à [2, 3, 4])

\subsection{Le cour et les vaisseaux}

La principale mission du système cardio-vasculaire (SCV), la fourniture d'oxygène et de nutriments aux différents tissus de l'organisme, est assurée par une double circulation sanguine, systémique (du cœur vers les tissus par les artères et retour par les veines) et pulmonaire (du cœur vers le poumon et retour), chacun de ces deux compartiments étant mis en mouvement par la pompe cardiaque correspondante (ventricule gauche ou droit). Cette double pompe est contrôlée, en fréquence et en volume éjecté à chaque battement, par le système nerveux autonome (SNA).

La fréquence cardiaque, principal facteur d'adaptation du débit, est donnée par un pacemaker naturel soumis au contrôle du SNA: le nœud sino-atrial, situé dans l'oreillette droite (il existe des modèles mathématiques au niveau de la cellule, dérivés du modèle nerveux de Hodgkin et Huxley, de ce pacemaker; ils ne seront pas rappelés ici, une revue sommaire pouvant en être trouvée dans [5]).

Le volume de sang éjecté dans la circulation systémique à chaque battement, ou volume d'éjection, dépend de 3 facteurs : a/ la précharge, ou volume de sang présent dans le ventricule gauche juste avant l'éjection (volume dépendant de la pression du retour veineux au cœur, dite pression veineuse centrale,

*INRIA Rocquencourt

†Université Paris VIII et INRIA Rocquencourt 
PVC); b/ la contractilité du myocarde, sous la dépendance du SNA; c/ la postcharge, ensemble des facteurs qui s'opposent au travail du cœur: résistance pariétale du ventricule, impédance de l'aorte, et résistances périphériques, ces dernières étant également sous la dépendance du SNA.

Le circuit vasculaire n'est pas seulement pourvu de résistances, il est aussi pourvu de capacités. Les vaisseaux sanguins ne sont en effet pas rigides, mais compliants, et les veines systémiques, notamment, constituent un important réservoir de sang. Les quatre compartiments du circuit vasculaire : artériel et veineux systémique, artériel et veineux pulmonaire sont ainsi modélisables en première intention chacun par une cellule du premier ordre (ou éventuellement du second ordre, si l'on prend en compte les effets d'inertie du sang). La principale variable d'état de chacun de ces quatre compartiments est la pression sanguine, et celle qui joue le rôle le plus important est la plus élevé : la pression artérielle systémique.

\subsection{Contrôle de la pression systémique par le SNA}

Les barorécepteurs artériels sont sensibles à l'étirement de la paroi ; Ils transmettent des messages nerveux, sous la forme de trains d'impulsions électriques, codés en fréquence, par des fibres afférentes (= de la périphérie vers le centre) qui ont leur terminaison dans le tronc cérébral.

Les barorécepteurs du circuit veineux sont situés dans l'oreillette droite; ils envoient aux centres du tronc cérébral des messages qui ont pour effet d'adapter le contrôleur (le SNA) à la pression veineuse de retour et aux variations de la pression intrathoracique dues à la respiration.

Du tronc cérébral partent les deux branches efférentes du SNA, sympathique et parasympathique, qui ont des effets antagonistes sur la pression artérielle systémique: une stimulation sympathique augmente la fréquence cardiaque et la contractilité des fibres myocardiques, augmente aussi les résistances périphériques, et au total la pression, tandis qu'une stimulation parasympathique, principalement à destination du pacemaker sinusal, ralentit le cœur et fait chuter la pression.

Ces voies ont des temps d'action très différents : la voie sympathique, cardioaccélatrice, est efficace en une dizaine de battements cardiaques, tandis que le délai d'action du vague transmis par la voie parasympathique est très court, de l'ordre du battement cardiaque (on parle de "coup de frein vagal"). Leur action est de trois types: action sur la fréquence cardiaque, soit par modification du rythme du pacemaker, soit par modification de la vitesse de transmission de l'influx électrique dans la paroi du cœur; action sur la puissance de la pompe, soit directement sur la vitesse de contraction des fibres musculaires, soit sur la tension et la relaxation de ces fibres ; action enfin sur les résistances périphériques : vasoconstriction ou vasodilatation.

\section{Principes de modélisation de la circulation sanguine}

On présente dans ce qui suit un modèle mathématique de la pompe cardiaque inséré dans la circulation sanguine. L'ensemble est vu comme un système dynamique mécanique dont on étudie l'activité périodique, initiée par une commande chimique elle-même reliée au pacemaker électrique du nœud sinusal.

Un modèle simple de la géométrie du ventricule permet de relier les variables contrainte et déformation de la fibre aux variables pression et volume du ventricule. Le reste de la circulation est un ensemble de compartiments caractérisés par des constantes de temps de remplissage et de vidage. 
Ce modèle, parce qu'il repose sur des considérations physiques, rend compte de phénomènes qualitatifs clefs.

\subsection{Modèle de fibre musculaire}

On suppose un montage de type Hill-Maxwell ([3], [4]): les éléments contractiles (EC), actifs, sont en série avec des éléments élastiques linéaires passifs qui "absorbent" les déformations quand la fibre ne peut pas se déformer dans son ensemble (phases isométriques); le tout est en parallèle avec des éléments élastiques non linéaires (EP) passifs, qui assurent plutôt la cohésion de l'ensemble. La contrainte totale $\sigma$ dans la direction des fibres est la somme des contraintes de EC et de EP :

$$
\sigma=\sigma_{C}+\sigma_{P}
$$

On note $\varepsilon_{C}$ la déformation de EC et $\varepsilon$ celle de EP (aussi égale à la déformation totale).

\subsubsection{Lois de comportement}

\section{a/ Partie contractile :}

Les mesures de l'activité du muscle en général et du myocarde en particulier montrent que la force développée n'est pas proportionnelle à la déformation de la fibre. Nous proposons (voir [6]) le modèle :

$$
\dot{\sigma}_{C}=-k_{1} \sigma_{C}\left|\dot{\varepsilon}_{C}\right|+k_{2} \dot{\varepsilon}_{C}+k_{C} u
$$

où $k_{1}, k_{2}$, et $k_{C}$ sont des raideurs.

Si on fait $u=0$ dans (1) on obtient une relation contrainte-déformation élasto-plastique, qui fournit une loi de comportement satisfaisante pour le muscle (voir [7]).

De plus, les parties contractiles (sarcomères) sont actives : elles développent une force en réponse à une commande chimique (modification de la concentration en ions calcium intracellulaire). Le terme en $u$ en rend compte dans (1), il permet en particulier de "démarrer" la contraction à partir du repos. Ce modèle vaut pour la fibre entière (mise en série de sarcomères) qui, dans le cas du myocarde, est sollicitée périodiquement; nous proposons en première intention pour $u$ un "créneau" (fonction constante par morceaux, ne prenant que deux valeurs), périodique à la période cardiaque, pouvant correspondre par un intermédiaire chimique aux deux temps électriques (dépolarisation / repolarisation) de la membrane de la cellule excitable cardiaque.

\section{b/ Éléments passifs :}

L'élément passif parallèle contribue à augmenter la contrainte au-delà d'une déformation seuil (pour protéger le cœur des trop grands remplissages : il concourt à la fermeture de la valve quand la déformation devient trop grande). On propose la loi de comportement : $\sigma_{P}(\varepsilon)=k_{P}|\varepsilon-\bar{\varepsilon}|_{+}$, où $k_{P}$ est une raideur, et $\bar{\varepsilon}$ représente une déformation seuil.

\subsection{2 Équation du mouvement}

Des mesures réelles montrent que lors de la contraction et de la relaxation isovolumiques la paroi endocavitaire est soumise à une accélération importante qui a une signification physiologique (voir 
[8]). On en rend compte en écrivant la relation de la dynamique pour les éléments contractiles; on propose l'équation (2) dans laquelle on a ajouté un terme d'amortissement (frottement visqueux) et un autre de rappel élastique :

$$
\ddot{\varepsilon}_{C}+2 \zeta \omega \dot{\varepsilon}_{C}+\omega^{2} \varepsilon_{C}=-\alpha \sigma_{C}+\beta \varepsilon
$$

Les paramètres $\zeta, \omega, \alpha, \beta$ sont reliés à ceux, physiques, caractérisant la fibre (raideur, masse, longueur au repos).

\subsection{Modèle de ventricule}

On suppose un seul degré de liberté pour la déformation du cœur. Par exemple, on assimile le cœur à un cylindre de hauteur $a$, de rayon $b$ et d'épaisseur h. On suppose que la déformation a lieu uniquement suivant $a: a=a_{0}(1+\varepsilon)$ et $b=b_{0}$.

$V$ est le volume du cylindre; on a $V=V_{0}(1+\varepsilon)$ et donc $\dot{V}=V_{0} \dot{\varepsilon}, V_{0}$ étant le volume au repos.

Ce modèle fournit donc une relation simple entre la déformation de la fibre et celle de l'objet en trois dimensions.

On suppose dans un premier temps que l'épaisseur du ventricule est petite et constante: $h=h_{0}$.

La contrainte $\sigma$ est alors reliée à la pression dans le ventricule $P$ par l'intermédiaire de la relation de Laplace :

$$
P=\frac{2 h_{0}}{b_{0}} \sigma
$$

La pression interne est donc donnée, en notant $\bar{V}=V_{0}(1+\bar{\varepsilon})$ par (4):

$$
P\left(V, \sigma_{C}\right)=\frac{2 h_{0}}{b_{0}}\left[\sigma_{C}+\frac{k_{P}}{V_{0}}|V-\bar{V}|_{+}\right]
$$

\subsection{Le ventricule inséré dans la circulation}

On couple les équations (1) et (2) à l'équation de conservation du volume ventriculaire; par exemple, on obtient pour le ventricule gauche l'équation (5):

$$
\dot{V}=\frac{1}{R_{p v}}\left|P_{p v}-P\left(V, \sigma_{C}\right)\right|_{+}-\frac{1}{R_{s a}}\left|P\left(V, \sigma_{C}\right)-P_{s a}\right|_{+}
$$

où $P\left(V, \sigma_{C}\right)$ est donnée par (4).

$R_{p v}$ et $R_{s a}$ sont les résistances à l'écoulement du sang à travers les valves cardiaques respectivement veineuse (mitrale) et artérielle (sigmoïdes aortiques).

D'autre part les pressions $P_{p v}(t)$ et $P_{s a}(t)$ désignent respectivement les pressions pulmonaire veineuse et systémique artérielle. Ce sont des variables d'état du système complet; par exemple, l'évolution de la pression artérielle systémique est régie par l'équation différentielle (6) :

$$
C_{s a} \dot{P}_{s a}=\frac{1}{R_{s a}}\left|P\left(V, \sigma_{C}\right)-P_{s a}\right|_{+}-\frac{1}{R_{S}}\left(P_{s a}-P_{s v}\right)
$$

où $P_{s v}(t)$ est la pression systémique veineuse; $C_{s a}$ est la compliance artérielle systémique et $R_{S}$ est la résistance totale systémique.

On pourra se référer à [9] pour la vision schématisée (par compartiments) de la boucle complète de circulation du sang. 


\subsection{Comportements qualitatifs}

On présente une liste non exhaustive de comportements qui servent à tester qualitativement le modèle.

\subsubsection{Contraction isotonique}

Par définition de la contraction isotonique, $\dot{\sigma}_{C}=0$, avec $\dot{\varepsilon}_{C}<0$, alors (1) donne

$$
\dot{\varepsilon}_{C}=-\frac{k_{C}}{k_{1} \sigma_{C}+k_{2}} u
$$

Cette équation rend compte de résultats expérimentaux selon lesquels le muscle se raccourcit d'autant moins vite qu'il doit équilibrer une charge plus importante (voir p. ex. [3]). Si cette charge est nulle, le muscle se raccourcit à la vitesse maximale $v_{\max }$ qui, d'après (7), vaut $-\frac{k_{C}}{k_{2}} u$.

À l'échelle du sarcomère la physiologie relie $v_{\max }$ aux taux d'hydrolyse de l'ATP par le complexe Actine-Myosine ([4]) ; ceci donne donc une information sur l'amplitude de $u$ durant sa phase positive.

\subsubsection{Fonctionnement de la pompe et loi de Starling}

Quand le cœur se remplit, son volume $V$ augmente, ce qui se traduit par une augmentation de $\varepsilon$. Donc, d'après (4), à partir d'un certain volume de remplissage, $P$ augmente, jusqu'à dépasser la pression dans le compartiment veineux, ce qui provoque la fermeture de la valve (voir (5)). À cet instant $P$ est égale à la précharge. Puis $P$ continue d'augmenter, maintenant grâce à $\sigma_{C}$, jusqu'à atteindre la pression dans le compartiment artériel: postcharge (voir [2] pour une introduction à ces notions, et [4] pour une description détaillée).

Si on élargit le cadre au contexte non pacemaker, on peut donc constater que : plus le cœur aura admis de sang - sous l'effet d'une pression veineuse importante et/ou d'une pression intraventriculaire de début de remplissage faible - plus la composante passive de $P$ contribuera à l'éjection, i.e. à atteindre la pression dans le compartiment artériel. Comme le débit de sortie est fonction de cette différence de pression de part et d'autre de la valve (voir (5)), le volume éjecté en sera d'autant plus important. À cet effet peut s'ajouter celui d'une contractilité augmentée (inotropisme positif) à travers la commande chimique sur $\sigma_{C}$.

\subsubsection{Contraction et relaxation isovolumiques}

En situation isométrique les équations (1) et (2) forment un système isolé de la circulation extérieure, que l'on peut raccrocher à des grandeurs discrètes caractéristiques d'un cycle cardiaque : pendant la contraction isovolumique le volume intraventriculaire $V$ est constant et vaut le volume télédiastolique; il reflète donc la précharge. Pendant la relaxation isovolumique $V$ est égal au volume télésystolique, qui est l'image de la postcharge.

\subsection{Modèle de la circulation complète}

On donne les équations du système dynamique complet, dont chacun des paramètres a été introduit précédemment pour le ventricule gauche. 


\subsubsection{Ventricule gauche}

$$
\left\{\begin{array}{l}
\dot{\sigma}_{C_{G}}=-k_{1_{G}} \sigma_{C_{G}}\left|\dot{\varepsilon}_{C_{G}}\right|-k_{2_{G}} \dot{\varepsilon}_{C_{G}}+k_{C_{G}} u \\
\ddot{\varepsilon}_{C_{G}}=-2 \xi_{G} \omega_{G} \dot{\varepsilon}_{C_{G}}-\omega_{G}^{2} \varepsilon_{C_{G}}-\gamma_{G} \sigma_{C_{G}}+\delta_{G} \frac{V_{G}-V_{0 G}}{V_{0 G}} \\
\dot{V}_{G}=\frac{1}{R_{p v}}\left|P_{p v}-P_{G}\left(V_{G}, \sigma_{C_{G}}\right)\right|_{+}-\frac{1}{R_{s a}}\left|P_{G}\left(V_{G}, \sigma_{C_{G}}\right)-P_{s a}\right|_{+}
\end{array}\right.
$$

où $P_{G}\left(V_{G}, \sigma_{C_{G}}\right)=\frac{2 h_{0_{G}}}{b_{0_{G}}}\left[\sigma_{C_{G}}+\frac{k_{P_{G}}}{V_{0_{G}}}\left|V_{G}-\bar{V}_{G}\right|_{+}\right]$

\subsubsection{Ventricule droit}

$$
\left\{\begin{array}{l}
\dot{\sigma}_{C_{D}}=-k_{1_{D}} \sigma_{C_{D}}\left|\dot{\varepsilon}_{C_{D}}\right|-k_{2_{D}} \dot{\varepsilon}_{C_{D}}+k_{C_{D}} u \\
\ddot{\varepsilon}_{C_{D}}=-2 \xi_{D} \omega_{D} \dot{\varepsilon}_{C_{D}}-\omega_{D}^{2} \varepsilon_{C_{D}}-\gamma_{D} \sigma_{C_{D}}+\delta_{D} \frac{V_{D}-V_{0 D}}{V_{0 D}} \\
\dot{V}_{D}=\frac{1}{R_{s v}}\left|P_{s v}-P_{D}\left(V_{D}, \sigma_{C_{D}}\right)\right|_{+}-\frac{1}{R_{p a}}\left|P_{D}\left(V_{D}, \sigma_{C_{D}}\right)-P_{p a}\right|_{+}
\end{array}\right.
$$

où $P_{D}\left(V_{D}, \sigma_{C_{D}}\right)=\frac{2 h_{0_{D}}}{b_{0_{D}}}\left[\sigma_{C_{D}}+\frac{k_{P_{D}}}{V_{0_{D}}}\left|V_{D}-\bar{V}_{D}\right|_{+}\right]$

\subsubsection{Circuit vasculaire}

$$
\begin{aligned}
& \left\{\begin{aligned}
C_{s a} \dot{P}_{s a} & =\frac{1}{R_{s a}}\left|P_{G}-P_{s a}\right|_{+}-\frac{1}{R_{S}}\left(P_{s a}-P_{s v}\right) \\
C_{s v} \dot{P}_{s v} & =-\frac{1}{R_{s v}}\left|P_{s v}-P_{D}\right|_{+}+\frac{1}{R_{S}}\left(P_{s a}-P_{s v}\right) \\
C_{p a} \dot{P}_{p a} & =\frac{1}{R_{p a}}\left|P_{D}-P_{p a}\right|_{+}-\frac{1}{R_{P}}\left(P_{p a}-P_{p v}\right)
\end{aligned}\right. \\
& \text { avec } C_{p v} P_{p v}(t)=C_{s a} P_{s a}(0)+C_{s v} P_{s v}(0)+C_{p a} P_{p a}(0)+C_{p v} P_{p v}(0) \\
& -\left[C_{s a} P_{s a}(t)+C_{s v} P_{s v}(t)+C_{p a} P_{p a}(t)\right]+V_{T} \\
& -\left[V_{G}(t)+V_{D}(t)+V_{s a}(0)+V_{s v}(0)+V_{p a}(0)+V_{p v}(0)\right]
\end{aligned}
$$

On a exprimé ici que sur les échelles de temps considérées le volume total $V_{T}$ est constant. 


\section{Modélisation du baroréflexe et mesures chez l'homme}

Le système nerveux autonome joue un rôle pivot dans la régulation à court terme de la pression artérielle via l'arc baroréflexe. Celui-ci, de façon très simplifiée, peut être modélisé comme sur la fig. 1, qui met en évidence les principaux mécanismes de contrôle de la pression artérielle par le SNA.

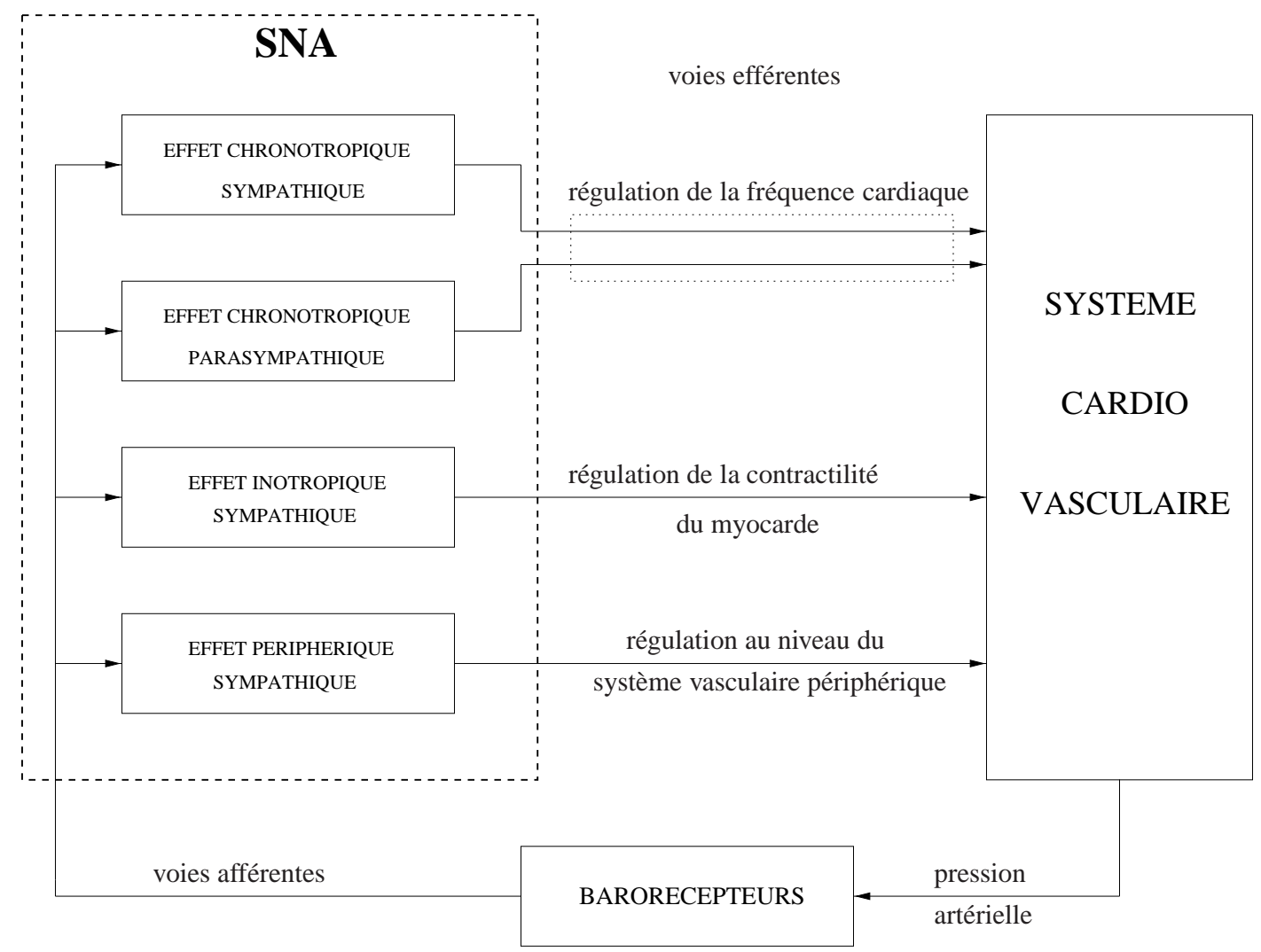

FIG. 1 - Arc baroréflexe: schéma de la régulation de la pression artérielle. Le SNA peut être vu comment le contrôleur. Quatre différents mécanismes de contrôle, divisés en parasympathique et sympathiques, ont été inclus dans le modèle.(voir le texte pour une brève introduction; pour une description complète du modèle voir [10] ou [11]).

Le modèle qui a été développé est un modèle en boucle fermée caractérisé par les éléments classiquement présents dans une boucle de contrôle :

- les capteurs et les voies afférentes

dans le modèle, ils ont été réduits simplement aux barorécepteurs à haute pression. On ne considère ni les récepteurs à basse pression ni l'intégration des informations au niveau central.

- mécanismes de contrôle de la pression artérielle

- réflexe chronotropique : contrôle par les branches efférentes antagonistes du SNA du rythme cardiaque ;

- réflexe inotropique : contrôle par la voie sympathique de la contractilité du myocarde ;

- réflexe vasculaire périphérique : contrôle par la voie sympathique du calibre des vaisseaux périphériques ; 


\section{- organes effecteurs}

- le pacemaker cardiaque: le SNA, agissant sur le nœud sinusal, est capable de régler la fréquence de contraction du muscle cardiaque;

- la contractilité du myocarde : le SNA peut agir sur la puissance de la pompe cardiaque ;

- les résistances périphériques : l'augmentation de l'activité du nerf sympathique sur le système vasculaire périphérique induit une vasoconstriction qui se traduit par une augmentation de ces résistances.

Le modèle a été développé dans [10] et repris dans [11] ; il reproduit les principales caractéristiques du système cardiovasculaire et ses réponses aux stimuli extérieurs : en particulier il reproduit l'Arythmie Sinusale Respiratoire (ASR) et l'apparition d'une composante en basse fréquence grâce à la présence d'un comportement résonnant autour de $0.1 \mathrm{~Hz}$ (ondes de Mayer). Des développements récents du modèle visent à rendre mieux compte d'autres phénomènes : entraînement des basses fréquences par la respiration, réponse du système cardiovasculaire à un changement de posture (tilt-up test), évaluation de la fonction cardiaque (en particulier dans l'insuffisance cardiaque).

\subsection{Arc baroréflexe : pression artérielle - rythme cardiaque}

Le but est de décrire, par de simples indicateurs numériques, l'interaction entre la pression artérielle (PA) et le rythme cardiaque (signal RR, suite des délais entre battements cardiaques successifs, détectés sur l'électrocardiogramme). De tels indicateurs doivent permettre d'évaluer le fonctionnement du SNA, à l'etat physiologique comme dans les états pathologiques. De ce point de vue, il est licite de simplifier le modèle, de façon à mettre en évidence dans l'arc baroréflexe seulement le contrôle du RR par le SNA (fig. 2). Sur cette figure est représentée l'interaction entre le centre respi-

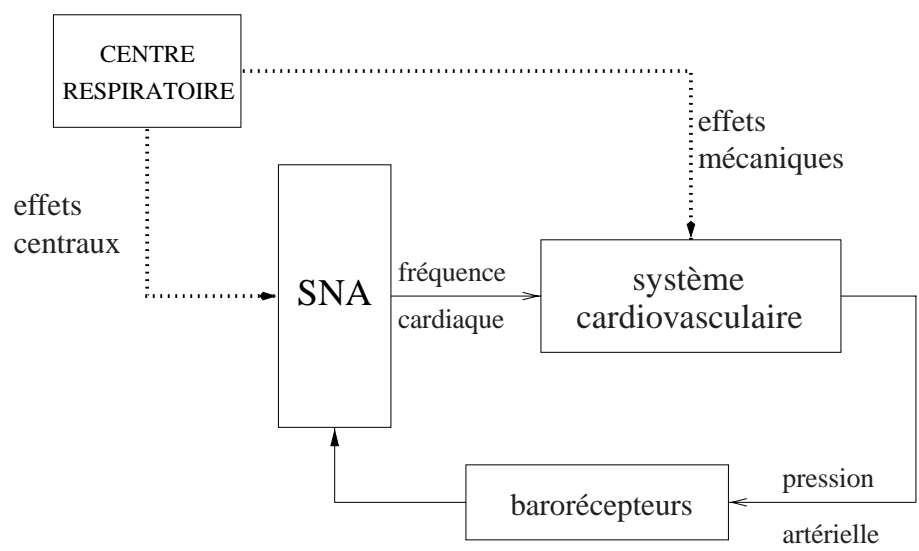

FIG. 2 - Schéma simplifié de l'arc de contrôle du rythme cardiaque $(R R)$, et des interactions aux deux niveaux, central et mécanique, de la respiration et du système cardio-vasculaire.

ratoire et le système cardiovasculaire : d'un côté une interaction au niveau central (interaction directe entre les centres autonomes bulbaires qui contrôlent le cœur et le centre respiratoire bulbaire), de l'autre, une interaction située plutôt au niveau mécanique (intrathoracique), due à la modification du retour veineux à l'inspiration. 


\subsubsection{Point de vue entrée-sortie}

Pour évaluer les influences de la PA sur le RR, le système étant en boucle fermée, on doit supposer faibles les interactions centrales entre le centre respiratoire et le centre autonome qui règle le rythme cardiaque. Sous cette hypothèse, on se place dans un contexte de relation entrée - sortie, où l'entrée est la PA et la sortie le RR. Il devient naturel d'étudier conjointement les variations des deux signaux pour bien interpréter le fonctionnement du contrôleur.

\subsubsection{Le rôle de la respiration}

De nombreuses recherches ont démontré que la fréquence et le volume respiratoires ont une large influence sur la variabilité du RR et de la PA. Pour que les fluctuations de l'intervalle RR puissent être considérées comme des indicateurs fiables du contrôle nerveux, en particulier quand l'ASR est utilisée pour quantifier le tonus vagal, il faut donc faire en sorte que les interactions entre la respiration et le système nerveux autonome soient constantes durant chaque phase de mesures cliniques. Ainsi dans l'expérience qui sera décrite ci-dessous, nous avons fixé la fréquence respiratoire (imposant aux sujets de rythmer leur respiration à l'aide d'un métronome), de façon à obtenir une bande bien définie en haute fréquence (HF), la même pour tous les sujets.

\subsubsection{Analyse spectrale}

L'analyse spectrale de la variabilité du RR et de la PA, et leur étude conjointe par l'ananalyse interspectrale constituent des outils complémentaires d'analyse du SNA. Si $x_{i}$ et $y_{i}$ sont les i-ème valeurs du RR et de la PA (systolique, par exemple) on peut calculer les variables suivantes:

$$
\begin{array}{cccc}
\text { Transformée de Fourier Discrète } & X(k \Delta f) & = & \sum_{i=0}^{N-1} x_{i} w_{i} e^{-j 2 \pi k \Delta f i T_{o}} \\
\text { Densité spectrale d'énergie } & \bar{D}_{x x}(k \Delta f) & = & \frac{1}{M+1} \frac{1}{T} \sum_{k=-\frac{M}{2}}^{\frac{M}{2}}|X(k \Delta f)|^{2} \\
\text { Densité spectrale d'énergie croisée } & \bar{D}_{x y}(k \Delta f) & = & \frac{1}{M+1} \frac{1}{T} \sum_{k=-\frac{M}{2}}^{\frac{M}{2}} \tilde{X}(k \Delta f) Y(k \Delta f) \\
\text { Module de la fonction de transfert } & \left|H_{x y}(k \Delta f)\right|= & \frac{\left|\bar{D}_{x y}(k \Delta f)\right|}{\bar{D}_{x x}(k \Delta f)} \\
\text { Phase de la fonction de transfert } & \Phi_{x y}(k \Delta f) & = & \tan ^{-1}\left(\frac{i \operatorname{mag}\left[\bar{D}_{x y}(k \Delta f)\right]}{\operatorname{real}\left[\bar{D}_{x y}(k \Delta f)\right]}\right) \\
\text { Fonction de Cohérence } & \gamma_{x y}^{2}(k \Delta f) & = & \frac{\left|\bar{D}_{x y}(k \Delta f)\right|^{2}}{\bar{D}_{x x}(k \Delta f) \bar{D}_{y y}(k \Delta f)}
\end{array}
$$

où $\tilde{X}(k \Delta f)$ est le conjugué de $X(k \Delta f), T_{o}$ est le pas d'échantillonage en temps, $\Delta f$ est le pas d'échantillonnage en fréquence et $w_{i}$ est la i-ème composante de la fenêtre de lissage. Pour évaluer l'état du système nerveux autonome, on calcule les paramètres suivants dans chaque fenêtre fréquen- 
tielle, en basse (description des ondes de Mayer) et en haute fréquence (liée à l'activité respiratoire) :

$$
\begin{array}{clc}
\text { Énergie } & x_{* F} & =\sum_{k \Delta f \in * F} \bar{D}_{x x}(k \Delta f) \\
\text { Cohérence moyenne dans la fenêtre } & c o h_{* F} & =\frac{1}{\mathcal{L}_{* F}} \sum_{k \Delta f \in * F} \gamma_{x y}^{2}(k \Delta f) \\
\text { Gain moyen dans la fenêtre } & g_{* F}=\frac{1}{\mathcal{L}_{* F}} \sum_{k \Delta f \in * F}\left|H_{x y}(k \Delta f)\right|
\end{array}
$$

où la bande de fréquence $* F$ peut être HF (haute fréquence) ou LF (low frequency, i.e. basse fréquence), $\mathcal{L}_{* F}$ est la largeur de la fenêtre considérée. Chaque signal cardiovasculaire a donc été caractérisé par son contenu énergétique en haute et en basse fréquence.

\subsubsection{Proposition d'indicateurs pour l'évaluation de l'arc baroréflexe}

On introduit ici deux autres indicateurs, déduits d'une analyse du modèle de l'arc baroréflexe, et qui vont décrire l'état du fonctionnement du SNA :

$$
\text { Gain normalisé }: \quad \bar{g}_{* F}=g_{* F} \frac{x_{\text {mean }}}{y_{\text {mean }}}
$$

Index de sensibilité : $\frac{\bar{g}_{L F}}{g_{H F}}$

Ces indicateurs peuvent être calculés pour chaque bande de fréquence (ce qui à notre connaissance n'avait encore jamais été fait), l'index de sensibilité permettant alors la comparaison directe entre le comportement du contrôleur en haute et en basse fréquence séparément. Le gain a été normalisé par les valeurs moyennes du RR et de la PA, ce qui permet de prendre en compte le point de consigne (en pression) du système cardiovasculaire.

\subsubsection{Résultats expérimentaux}

Les changements du SNA provoqués par un stimulus extérieur (tilt-test) ont été évalués en examinant les signaux cardio-vasculaires et respiratoire. On s'est limité à l'étude des périodes stationnaires, et on a donc exclu de l'analyse les périodes qui suivent des changements de respiration (spontanée versus contrôlée) ou de posture (allongée versus debout).

Le protocole est représenté sur la figure 3, il se décompose en 3 étapes successives : 5 minutes $(\mathrm{mn})$ de respiration spontanée en position allongée; $5 \mathrm{mn}$ de respiration contrôlée à $0.25 \mathrm{~Hz}$ (on contrôle seulement la fréquence respiratoire, pas le volume) et finalement, après un changement de posture (tilt-test passif), $5 \mathrm{mn}$ en gardant la fréquence respiratoire contrôlée à $0.25 \mathrm{~Hz}$.

On constate (fig. 4) en basse fréquence (LF) une augmentation importante de l'énergie pour la PAS, et une augmentation moins évidente pour le RR. L'énergie en haute fréquence (HF) disparaît pour le RR (inhibition parasympathique) tandis qu'elle augmente pour la PAS (effet mécanique de la respiration plus important). 
Tilt $60^{\circ}$

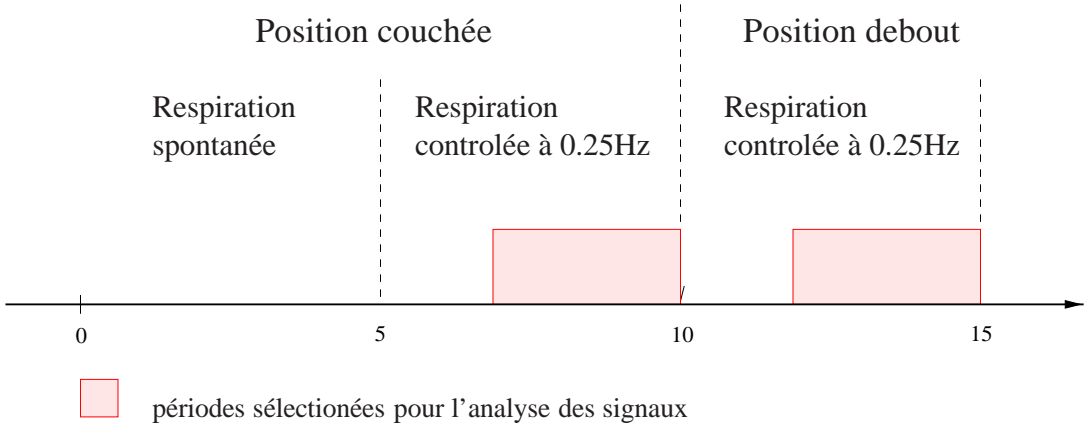

FIG. 3 - protocole expérimental utilisé pour étudier le SNA et la réponse du système cardiovasculaire à des changements de position.

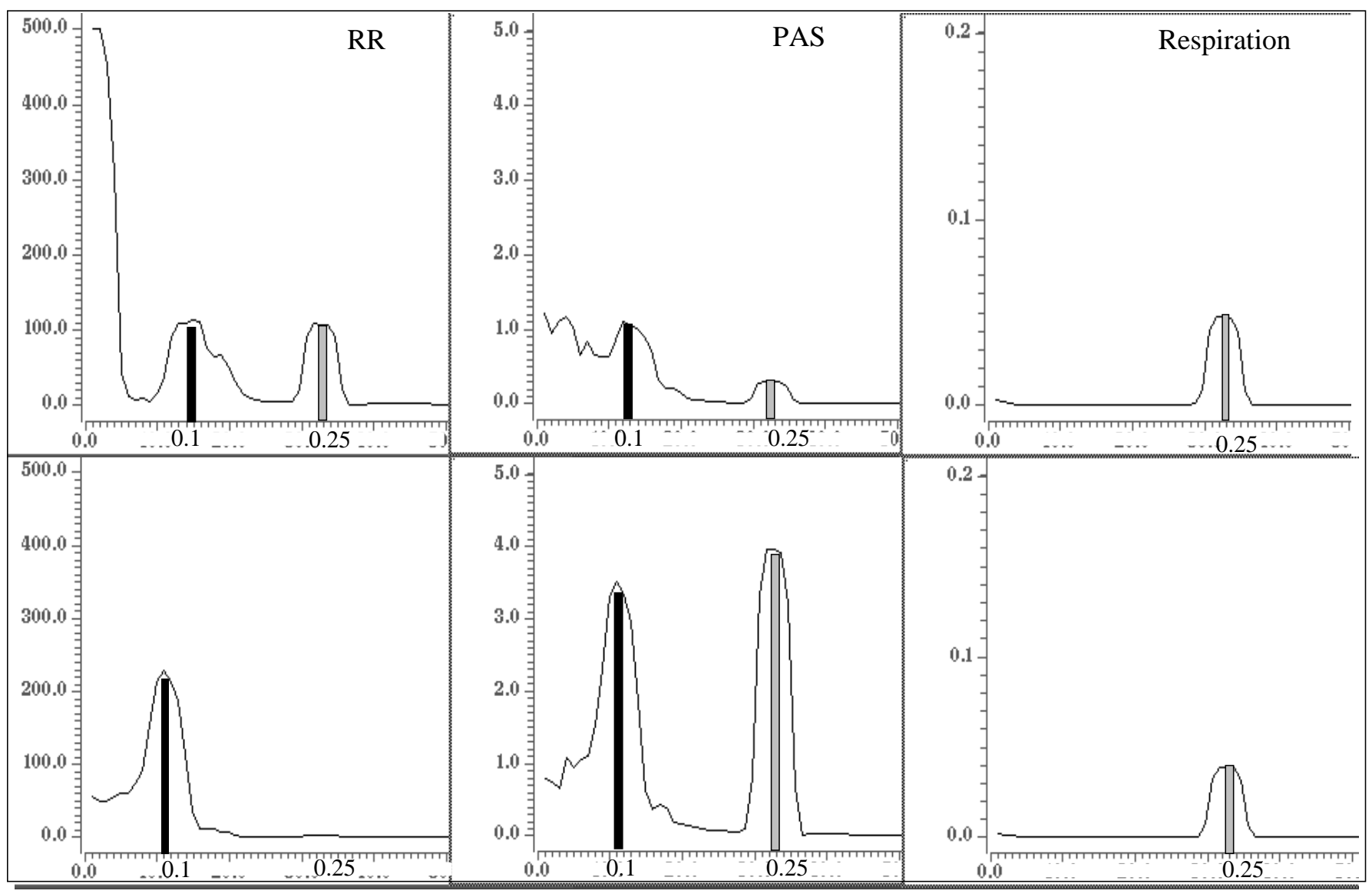

FIG. 4 - Variations des composantes spectrales des signaux cardio-vasculaires induites par tilt-test. En haut en position couché, en bas en position debout. En abscisses sont représentées les fréquences et en ordonnées la puissance spectrale [ $\mathrm{ms}^{2} / \mathrm{Hz}$ ] pour le rythme cardiaque (RR), [ $\mathrm{mmHg}^{2} / \mathrm{Hz}$ ] pour la pression artérielle systolique (PAS), et pour la respiration [en unités arbitraires]. L'énergie en basse fréquence est représentée par une barre verticale noire, celle en haute fréquence par une barre grise. 
Comme on peut le remarquer à l'aide d'une analyse temps-fréquence, ou en comparant les résultats obtenus par DFT (Transformation de Fourier Discrète) sur plusieurs fenêtres d'analyse, les composantes en basse fréquence du RR et de la PAS présentent un comportement en bouffées, bien visible sur la figure 5 (cette figure inclut toutes les périodes, sans limitation aux périodes stationnaires).
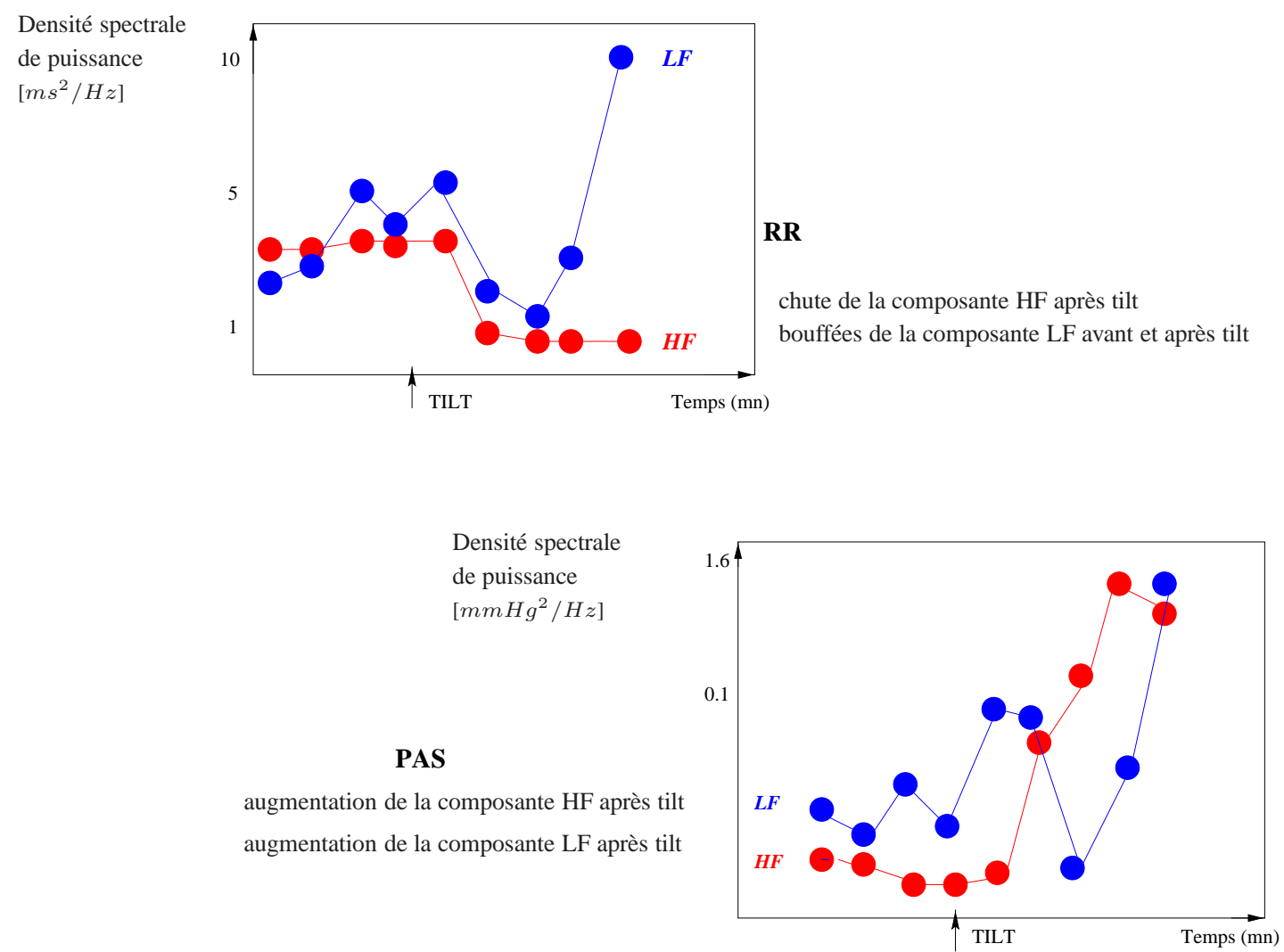

FIG. 5 - Évolution temporelle de l'énergie en basse $(L F)$ et haute fréquence $(H F)$ des signaux rythme cardiaque $(R R)$ et pression systolique (PAS) avant et après tilt. La composante spectrale en basse fréquence présente des bouffées, tandis que celle en haute fréquence est plus stable, mais subit en revanche un grand changement lors du tilt-test. Les spectres sont obtenus par DFT, sur des périodes de 2 minutes, avec recouvrement de $50 \%$.

Même si la DFT n'est pas nécessairement le meilleur outil pour étudier le système cardiovasculaire, puisque son emploi suppose la stationnarité des signaux (et il est possible que les $5 \mathrm{mn}$ suivant le changement de posture ne soient pas suffisantes pour atteindre une situation stationnaire), on peut tenter d'interpréter l'instabilité de la composante en basse fréquence qui se manifeste ici par l'apparition de bouffées comme le reflet de la mise en jeu du régulateur : le SNA.

La figure 6 représente l'évolution temporelle des gains et de l'index de sensibilité proposé. Le gain en basse fréquence $g_{L F}$ évolue par bouffées et sans différences significatives avant et après tilt. Le gain en haute fréquence $g_{H F}$ décroît significativement, entraînant une chute de l'index de sensibilité.

Cette étude confirme la nécessité d'analyser conjointement les composantes en haute et basse fréquence du rythme cardiaque et de la pression artérielle, et surtout de décrire le contrôleur par des indicateurs liés aux gains et aux points de consigne du système cardiovasculaire. 


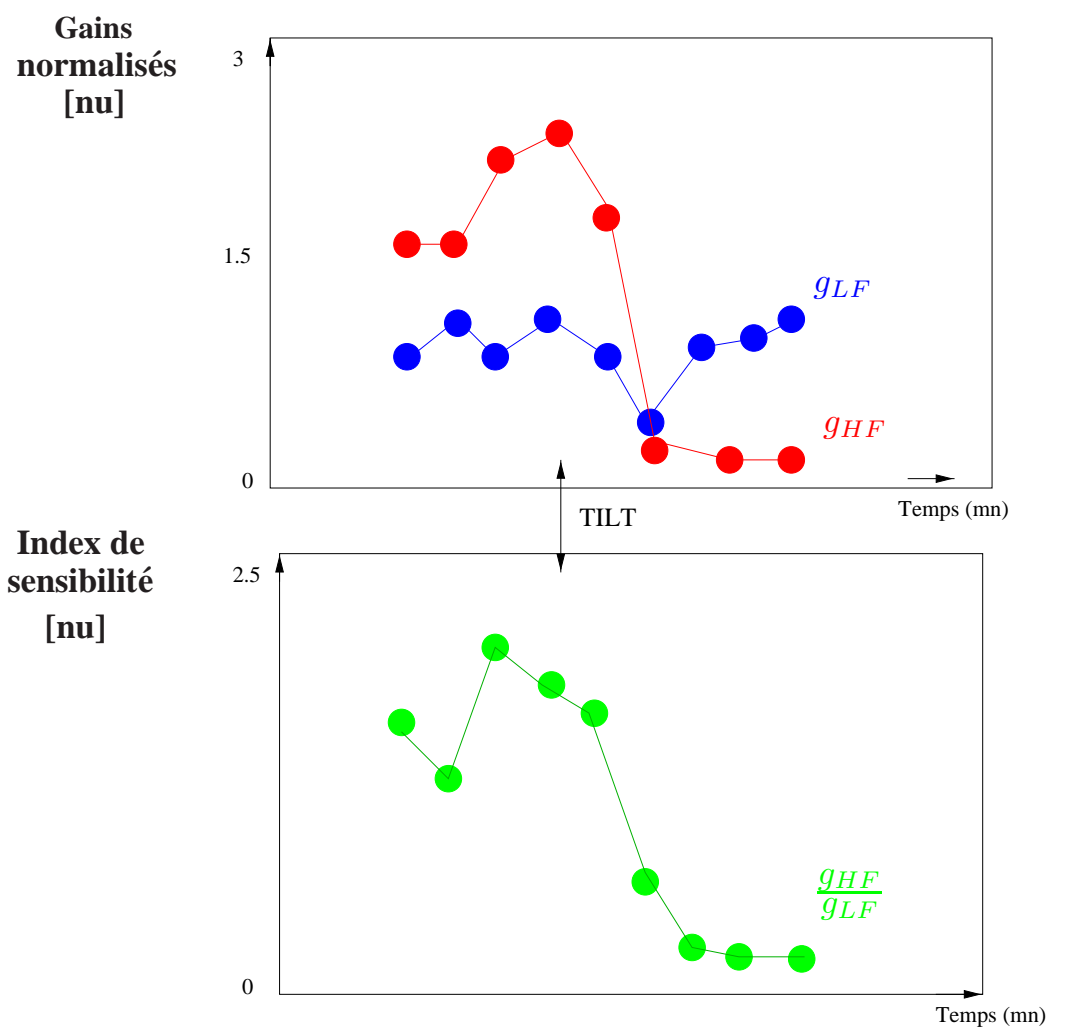

FIG. 6 - Évolution temporelle des gains normalisés en basse $\left(g_{L F}\right)$ et en haute $\left(g_{H F}\right)$ fréquence et de l'index de sensibilité après un stimulus postural. Les spectres sont obtenus par DFT, par périodes de 2 minutes avec recouvrement de 50\%. 


\section{Perspectives de la modélisation et mesures en clinique}

On a présenté ici deux aspects du travail de modélisation en cours : le muscle cardiaque vu comme un système dynamique commandé de manière périodique, d'une part, et l'arc baroréflexe, vu comme une fonction de transfert non linéaire, d'autre part. Beaucoup reste à faire pour ajouter à cet édifice d'autres sous-systèmes, et enfin pour les intégrer en un modèle simple, dont les paramètres soient identifiables et utilisables en routine par le clinicien.

\subsection{Nouvelles tâches de modélisation}

Dans les tâches entreprises, ou à entreprendre, pour obtenir un modèle du système cardio-vasculaire et respiratoire intégrant les divers sous-systèmes impliqués, on peut relever principalement :

- la modélisation simplifiée de la commande chimique musculaire à partir de la dépolarisation électrique membranaire des cellules ventriculaires;

- l'introduction d'un couplage électrique entre cellules pacemaker (du nœud sinusal) et non pacemaker (ventriculaires) pour prendre en compte le délai (variable) de transmission de l'influx électrique à l'intérieur de la paroi cardiaque (de tels modèles existent, cf. par exemple [12]) ;

- la prise en compte des effets d'inertie du sang dans les compartiments vasculaires, en particulier dans la circulation veineuse systémique: actuellement, ces compartiments sont conçus comme des cellules du premier ordre, un ordre 2 peut s'avérer nécessaire, en particulier lors de la mise en jeu du SCV à l'exercice physique ;

- la modélisation mécanique des barorécepteurs (transducteurs mécano-électriques) sur lesquels on ne possède à notre connaissance actuellement que des résultats fonctionnels, conduisant à des modèles du type $p+\alpha p^{\prime}=k\left(\nu+\beta \nu^{\prime}\right)$, où $p$ est la pression artérielle et $\nu$ la fréquence de décharge du nerf efférent [13], mais dans lesquels la base physiologique est questionnable;

- l'inclusion d'un modèle d'oscillateur respiratoire, et, sur le même principe, d'un modèle de centre nerveux autonome, et de leurs interactions centrales: de ce point de vue, les travaux de J. Champagnat et J.-F. Vibert exposés dans le présent volume sont une avancée importante dans cette voie;

- à chacun des étages où la régulation par le SNA est mise jeu, l'introduction du contrôle nerveux comme une commande venant remplacer un paramètre considéré comme fixe dans le soussystème cible; en particulier:

- modélisation des effets chronotropes: contrôle par le SNA de la période de la cellule pacemaker du nœud sinusal (voir p. ex. [14]);

- modélisation des effets dromotropes : contrôle par le SNA du temps de conduction électrique intracardiaque (retard variable);

- modélisation des effets inotropes : contrôle par le SNA des paramètres de la commande chimique du muscle cardiaque, en particulier de la concentration en ions calcium intracellulaires ;

- modélisation des muscles circulaires lisses des résistances périphériques et de leur commande électrique par les rameaux sympathiques (modèle vraisemblablement du même type que celui du muscle cardiaque, avec bien sûr des paramètres différents). 


\subsection{Identification de paramètres par de nouvelles mesures cliniques}

Parallèlement à ce travail de modélisation, un travail de traitement et d'exploitation de mesures est en cours, dans le but immédiat d'identifier des paramètres des sous-modèles du SCV (et du SNA) considérés. Ces mesures, invasives ou non, sont réalisées par les équipes médicales avec lesquelles nous collaborons.

\subsection{Proposition de nouveaux indicateurs de fonctionnement du SCV et du SNA}

L'objectif de ce travail de modélisation, qui s'appuie pour l'identification des paramètres du modèle sur des campagnes de mesures cliniques, est en effet à terme de fournir aux praticiens des indicateurs de fonctionnement du SCV et de son contrôleur, le SNA :

- des indicateurs de la fonction cardiaque peuvent être obtenus à partir de mesures (a priori invasives) réalisées pendant les phases de contraction et de relaxation isovolumiques, en particulier par identification de caractéristiques de la commande $u$ et des paramètres du modèle de la fibre musculaire (cf. ci-dessus l'équation (1) dans "Modèle de la fibre musculaire"); dans ces phases, le sous-système mécanique considéré est en effet isolé et d'identification plus facile ;

- des indicateurs globaux du SCV considéré comme un système dynamique sont attendus des mesures portant sur les sorties telles que pression artérielle, rythme cardiaque et respiration;

- enfin des indicateurs portant sur le contrôleur à court terme (le SNA) devraient fournir aux cliniciens des renseignements permettant d'évaluer par exemple la stabilité de ce contrôleur, et éventuellement de diagnostiquer et de corriger certains troubles tels que hypotension orthostatique et syncope vasovagale, et certaines formes d'hypertension; un gain équivalent a déjà été proposé (cf. ci-dessus "Modélisation du baroréflexe et mesures chez l'homme") : il s'agirait ici notamment d'analyser l'adaptation du point de consigne du contrôleur.

\section{Références}

[1] Clairambault, J., Médigue, C., Bestel, J. ( 1997): Le système cardiovasculaire et sa régulation à court terme par le système nerveux autonome. In : Journées d'Étude "Automatique et Santé" du club EEA (organisateurs : E. Dombre et A. Fournier). ISIM, Montpellier, juin 1997.

(inclus en annexe à la fin de cet article)

[2] S. Silbernagl and A. Despopoulos. Atlas de poche de physiologie. Flammarion, Médecinesciences, 1995.

[3] G. Chauvet. Traité de Physiologie théorique : De la cellule à l'homme. Vol. 2, Masson, 1987.

[4] A. M. Katz. Physiology of the Heart. Raven Press, 1992.

[5] Clairambault, J., Claude, D. Systèmes dynamiques et biologie: quelques exemples. Séminaire T. I. P. E. sur les systèmes dynamiques. Journée de formation (ENSTA-UPS) pour les professeurs de Mathématiques Spéciales (organisateur: Max Bezard). ENSTA, Paris, mai 1996.

[6] Bestel, J., Sorine, M. Modélisation mathématique du système cardio-vasculaire. Rapport de Recherche INRIA, à paraître.

[7] I. Mirsky and W. W. Parmley. CARDIAC MECHANICS: Physiological, Clinical, and Mathematical Considerations. Chap. 4. 1974. 
[8] SORIN-BIOMEDICA. Documentation technique sur la sonde BEST.

[9] F. C. Hoppensteadt and C. S. Peskin. Mathematics in Medicine and the Life Sciences. Chap. 5. Springer Texts in Applied Mathematics, 1992.

[10] Vermeiren, C. Analyse et modélisation du système cardio-vasculaire et sa régulation à court terme par le système nerveux autonome. Thèse de doctorat en génie biologique et médical, Université Paris Val de Marne, décembre 1996.

[11] Monti, A. Parameter assessment in a model for the control of cardiovascular functions. Thèse de fin d'études, Politecnico di Milano, octobre 1998.

[12] Landau, M., Lorente, P., Henry, J., Canu, S. Hysteresis phenomena between periodic and stationary solutions in a model of pacemaker and nonpacemaker coupled cardiac cells. J. Math. Biol., 25:491-509, 1987.

[13] Taher, M., Cecchini, A.B.P., Allen, M.A., Gobran, S.R., Gorman, R.C., Guthrie, B.L., Lingenfelter, K.A., Rabbany, S.Y., Rolchigo, P.M., Melbin, J., Noordergraaf, A. Baroreceptor responses derived from a fundamental concept. Ann. Biomed. Eng., 16: 429-443, 1988.

[14] Clairambault, J. A model of the autonomic control of heart rate at the pacemaker cell level through G-proteins. 17e IEEE-EMBS, 1379-1380, Montréal, 1995. 


\title{
Annexe : Le système cardiovasculaire et sa régulation à court terme par le système nerveux autonome
}

\author{
J. Clairambault, C. Médigue, J. Bestel
}

Journées Automatique et Santé, Montpellier, mai 1997, Réf. [1] du présent article (1999)

\section{Résumé}

\begin{abstract}
On présente le système cardiovasculaire (SCV) dans son ensemble, avec ses compartiments : pompe cardiaque, petite et grande circulation, circuit artériel et circuit veineux, les variables observables en clinique, les mécanismes de contrôle, dont le système nerveux autonome (SNA), et les interactions du SCV avec la respiration. Les problèmes de modélisation sont abordés sous l'angle de la commande, c'est-àdire en fonction d'une mission à remplir pour le SCV et son contrôleur. Les possibilités de commande pharmacologique du SNA sont également examinées, et des applications à la clinique envisagées.
\end{abstract}

\section{Présentation du système cardiovasculaire (SCV)}

La principale mission du SCV est la fourniture d'oxygène et de nutriments aux différents tissus de l'organisme, en respectant une hiérarchie dans les distributions régionales : cerveau, puis rein, territoire splanchnique (celui de la digestion...), et membres. La sélection d'un territoire aux dépens des autres se fait par vasoconstriction (réduction du calibre des vaisseaux), dans les territoires négligés.

\subsection{Moyens mis en ouvre}

Les moyens mis en œuvre pour assurer cette mission sont une pompe: le cœur, et un circuit hydraulique qu'on peut considérer, du moins à court et même à moyen terme (c'est-à-dire indépendamment de la réabsorption rénale), à volume constant : le système vasculaire.

\subsubsection{Le cœur en tant que pompe}

La pompe cardiaque est un générateur de pression, ou plus exactement de débit (la pression étant le produit du débit par la résistance opposée au travail de la pompe : si on assimile la pression à un voltage et le débit à une intensité de courant, il ne s'agit ni plus ni moins que de la loi d'Ohm). Cette pompe est en fait elle-même séparée de manière étanche (séparation achevée à la naissance) en deux pompes, l'une à haute pression vers l'ensemble de l'organisme (le ventricule gauche), et l'autre à basse pression vers le poumon (le ventricule droit). L'égalisation des débits droit et gauche est réalisée par un mécanisme d'adaptation purement mécanique : l'effet Starling (voir ci-dessous : cahier des charges physiologiques). Cette double pompe a une fréquence et un volume d'éjection (les deux facteurs du débit cardiaque) variables ; ces deux facteurs sont en permanence contrôlés, en particulier par le système nerveux autonome (SNA).

\subsubsection{Pacemaker et conduction électrique}

La fréquence cardiaque est le principal facteur d'adaptation du débit. Elle est donnée par un pacemaker naturel, situé dans l'oreillette droite: le nœud sinusal, qui est soumis au contrôle du SNA. 
À partir du nœud sinusal l'influx électrique se propage (à vitesse variable, et cette vitesse est aussi soumise au contrôle du SNA) vers le myocarde ventriculaire, lieu du couplage excitation-contraction.

\subsubsection{Volume d'éjection}

Le volume d'éjection dépend de 3 facteurs : a/ la précharge, ou volume de sang présent dans le ventricule gauche juste avant l'éjection (volume proportionnel à la pression du retour veineux au cœur, dite pression veineuse centrale, PVC) ; b/ la contractilité du myocarde, sous la dépendance du SNA; c/ la postcharge, ensemble des facteurs qui s'opposent au travail du cœur: résistance pariétale du ventricule, impédance de l'aorte, et résistances périphériques.

\subsubsection{Les deux circulations}

Le circuit vasculaire est divisé en deux parties : la circulation dite systémique, ou grande circulation, qui apporte oxygène et nutriments aux tissus, et la circulation pulmonaire, ou petite circulation, qui assure la navette entre le cœur, générateur de débit, et le poumon, générateur d'oxygène. L'oxygène est la matière première de la chaîne de phosphorylation oxydative de la mitochondrie, centrale energétique de la cellule, et on peut définir la puissance consommée par un organe comme le produit du débit sanguin le traversant par la différence artérioveineuse, différence des concentrations en oxygène dans le sang artériel (à l'entrée de l'organe, à haute pression) et dans le sang veineux (à sa sortie, à basse pression). Le maintien d'une pression artérielle efficace, permettant à la circulation d'assurer une bonne oxygénation des tissus, est assuré par deux mécanismes complémentaires : la régulation du débit cardiaque et celle des résistances périphériques.

\subsubsection{Résistances périphériques}

L'ensemble des résistances périphériques, encore appelées shunts artérioveineux, constitue la résistance systémique, qu'on peut définir par la loi d'Ohm comme le quotient d'une différence de pression artérioveineuse globale (mesurée entre la sortie du cœur au niveau des valvules sigmoïdes aortiques et le retour veineux au niveau de l'abouchement de la veine cave) par le débit cardiaque. Ces résistances périphériques sont soumises principalement à deux types de régulations. L'une, dite autorégulation, est purement locale et a pour but de maintenir une fourniture en oxygène, c'est-à-dire un débit artériel local, efficace même lorsque la pression chute ; un modèle simple consiste à rendre cette résistance tissulaire locale proportionnelle (grâce à des chémorécepteurs) à la concentration veineuse en oxygène. L'autre est sous la dépendance du même contrôleur que le débit cardiaque (le SNA).

\subsubsection{Compliance vasculaire}

Le circuit vasculaire n'est pas seulement pourvu de résistances, il est aussi pourvu de capacités. Les vaisseaux sanguins ne sont en effet pas rigides, mais compliants, et une augmentation de pression se traduira par une augmentation de leur calibre. Les veines systémiques, notamment, constituent un important réservoir de sang (environ 3 litres, contre moins d'un litre pour les artères systémiques), et le circuit veineux, circuit à basse pression, est aussi appelé circuit capacitif, par opposition au circuit artériel, à haute pression, dit circuit résistif. Mais les artères présentent elles aussi une certaine compliance; en particulier la compliance de l'aorte se manifeste par un aspect bifide (“dicrote" : à 
deux sommets) de l'onde de pression aortique, due à la réflexion de l'onde de propagation sur les résistances périphériques, lorsque les valvules sigmoïdes sont fermées.

\subsection{Critères à optimiser par la commande du SCV}

Il est légitime d'imposer au contrôleur de minimiser le travail de la pompe cardiaque, c'est-à-dire essentiellement celui du ventricule gauche, la plus puissante des deux pompes, celle qui débite à haute pression. Mais les deux pompes sont en fait synchrones, et le débit de la pompe droite est égal à celui de la pompe gauche; on peut donc définir ce travail comme une valeur moyenne au cours du temps du produit du débit cardiaque par la différence de pression artérioveineuse globale (entre la veine cave à l'entrée et l'aorte à la sortie).

La principale variable régulée est la pression artérielle. Normalement comprise entre 70 (valeur minimale $=$ diastolique) et $140 \mathrm{~mm} \mathrm{Hg}$ (maximale $=$ systolique), elle maintient un débit cérébral (le territoire cérébral est prioritaire, son approvisionnement se faisant éventuellement aux dépens des autres) satisfaisant jusqu'à environ $60 \mathrm{~mm} \mathrm{Hg}$ de pression systolique, après quoi le débit s'effondre.

\subsection{Mécanismes de contrôle de la pression artérielle}

\subsubsection{La régulation à long terme est réalisée :}

- soit par modification du volume plasmatique au niveau rénal; il s'agit d'une régulation pour l'essentiel hormonale : système rénine-angiotensine-aldostérone, $\mathrm{ADH}$ (hormone antidiurétique, alias vasopressine), facteur atrial natriurétique, dont la cible est rénale, mais aussi également d'une régulation passive, conséquence directe des variations de pression artérielle dans le rein;

- soit par modification des résistances périphériques : régulation hormonale par action vasoconstrictrice directe de l'angiotensine II.

\subsubsection{La régulation à moyen terme}

C'est une régulation hormonale par les catécholamines circulantes (noradrénaline surtout), qui sont sécrétées par la médullosurrénale sous l'influence de centres cérébraux (zones pressogènes), activés par la chute du débit sanguin local (ischémie cérébrale), mais aussi en cas de stress, et ont une action vasoconstrictrice qui peut atteindre jusqu'à 6 fois celle obtenue par le contrôle nerveux.

\subsubsection{La régulation à court terme}

C'est une régulation uniquement nerveuse, assurée par une partie du système nerveux qui fonctionne à l'état basal de manière indépendante de la vie de relation: le système nerveux végétatif, ou système nerveux autonome (SNA).

Les centres du SNA reçoivent des influences externes en provenance de l'hypothalamus (température), et, au-delà, du système limbique ou du cortex (émotions, à effet parfois quasi-immédiat sur le rythme cardiaque). De plus, pendant le sommeil paradoxal, la substance réticulée ascendante activatrice du mésencéphale exerce sur ces centres d'importantes perturbations. On se contentera de décrire ci-dessous le fonctionnement du SNA à l'état de base, ce qui suppose un sujet vigile, ou en état de sommeil profond, et aussi déconnecté que possible de perturbations extérieures éventuelles. 


\section{Le contrôleur à court terme : le système nerveux autonome}

\subsection{Capteurs et voies afférentes}

Des barorécepteurs, sensibles à la distension, sont situés dans la paroi des artères au niveau de la crosse de l'aorte et du sinus carotidien (élargissement de la lumière de l'artère carotide, situé à la hauteur de la bifurcation entre carotides externe et interne), et aussi sur le circuit veineux de retour, au niveau de l'oreillette droite. Les barorécepteurs artériels sont sensibles à des variations de pression minimes, de l'ordre de quelques $\mathrm{mm} \mathrm{Hg}$. Ces barorécepteurs transmettent des messages nerveux, sous la forme de trains d'impulsions électriques, codés en fréquence, par des fibres afférentes $(=$ de la périphérie vers le centre) qui rejoignent les IX ${ }^{\mathrm{e}}$ et $\mathrm{X}^{\mathrm{e}}$ paires crâniennes pour se terminer dans le noyau du tractus solitaire (NTS, sous le plancher du $4^{\mathrm{e}}$ ventricule, dans le tronc cérébral).

Les barorécepteurs de l'oreillette droite, ainsi que des mécanorécepteurs pulmonaires stimulés comme eux pendant l'inspiration, envoient des messages au NTS qui ont pour effet d'adapter le SNA à la pression veineuse de retour et aux variations de la pression intrathoracique dues à la respiration.

\subsection{Centres de contrôle}

À l'intérieur du NTS, des neurones secondaires déchargent suivant une fréquence nettement plus basse que ceux provenant directement des barorécepteurs, et répondent par une augmentation de leur fréquence de décharge à une augmentation de pression au niveau des barorécepteurs. Cette dépendance peut être modélisée comme intégrant à la fois une pression moyenne et la dérivée de la pression.

C'est vraisemblablement dans le NTS qu'on peut trouver la première non-linéarité de l'arc baroréflexe artériel (= la boucle de contrôle de la pression artérielle par les barorécepteurs et le système nerveux autonome). Cette non-linéarité, placée entre une entrée constituée des trains d'impulsions électriques en provenance des barorécepteurs, et une sortie à destination du noyau ambigu et de l'aire pressive bulbaire, est assimilable à un relais tout-ou-rien, sans doute avec hystérésis.

Le NTS est relié à deux centres bulbaires, d'une part au noyau ambigu, d'où partent des efférences parasympathiques (= vagales) à destination du cœur, d'autre part à l'aire pressive bulbaire, d'où partent des efférences sympathiques à destination du cœur et des vaisseaux. Il existe des inhibitions intercentrales réciproques, qui permettent un couplage dès l'origine de ces deux voies.

\subsection{Voies efférentes : la voie (ortho)sympathique et la voie parasympathique}

Les deux branches efférentes du SNA ont des effets antagonistes: une stimulation sympathique augmente la fréquence cardiaque et la contractilité des fibres myocardiques, augmente aussi les résistances périphériques, tandis qu'une stimulation parasympathique, principalement à destination du pacemaker sinusal, ralentit le cœur.

Ces voies ont des temps d'action très différents: la voie sympathique, cardioaccélatrice, est efficace en en une dizaine de battements cardiaques, tandis que le délai d'action du coup de frein vagal transmis par la voie parasympathique est quasi instantané, de l'ordre du battement. En dehors d'une raison téléologique immédiatement compréhensible par tout conducteur de véhicule à moteur, il y a à ce fait plusieurs explications d'ordre anatomique : la voie parasympathique part directement du centre encéphalique pour ne faire relais que dans la paroi même du cœur (le neurone postganglionnaire est ainsi très court), tandis que la voie sympathique fait relais très tôt, dans les ganglions sympathiques 
paravertébraux, donc loin du cœur. Or les fibres préganglionnaires sont myélinisées, et transmettent les impulsions électriques beaucoup plus rapidement que les fibres postganglionnaires, qui ne le sont pas ou peu. D'autre part, la synapse ganglionnaire sympathique est à simple entrée pour des sorties multiples (environ 1 pour 20, d'où un codage en fréquence, mais aussi en amplitude si on considère la sommation des potentiels d'action sur plusieurs voies parallèles), contre un codage (1 pour 1), en fréquence pure pour la voie parasympathique, d'où une possibilité de perte en ligne sur la voie sympathique. Enfin, la synapse neuro-effectrice cardiaque a un neuromédiateur différent dans les deux voies: acétylcholine pour la parasympathique, noradrénaline pour la sympathique, et la synapse noradrénergique serait plus lente que la synapse acétylcholinergique.

Il résulte de ces considérations anatomiques qu'on peut considérer la voie sympathique comme une voie à retard par rapport à la voie parasympathique.

\subsection{Organes effecteurs du SNA}

\subsubsection{Le pacemaker cardiaque, principal effecteur du SNA}

Le nœud sinusal est constitué de plusieurs milliers de cellules qui sont synchronisées, mais sont toutes douées d'automatisme. Beaucoup de modèles à base d'équations différentielles (non linéaires) en ont été proposés, qui tous reposent sur des variantes du modèle de Hodgkin et Huxley (1952) pour le potentiel d'action de la cellule nerveuse (on en trouvera une brève revue dans [6]). Ces modèles ont l'inconvénient d'être gourmands en nombre de variables, rendant compte de courants ioniques différents, et de ne pas être observables facilement à l'échelle d'un individu.

Le mode d'action des deux voies antagonistes du SNA au niveau de la cellule pacemaker cardiaque est assez bien connu ; la modulation par la voie sympathique (neuromédiateur : noradrénaline) se traduit essentiellement par un redressement de la pente (sensiblement constante) de la phase de dépolarisation diastolique lente du potentiel d'action de cette cellule, réduisant la durée du cycle cardiaque ; la modulation parasympathique (neuromédiateur: acétylcholine) a l'effet opposé. Des inhibitions réciproques mettent en place un couplage local sympathique / parasympathique au niveau de la jonction neuroeffectrice, qui vient compléter le couplage central. Cet effet du SNA est dit chronotrope. Il existe aussi un effet dit bathmotrope, d'action sur l'excitabilité des cellules du tissu de conduction par variation de la période réfractaire, mais cet effet semble peu important au niveau macroscopique.

Le nœud sinusal bat, hors de toute influence du SNA, à un rythme propre d'environ 105 battements par minute (bpm); mais il est en permanence soumis à des tonus, sympathique et parasympathique, qui ramènent ce rythme autour de 70 bpm (il y a normalement un tonus vagal dominant).

\subsubsection{La conduction auriculoventriculaire}

Les cellules du nœud sinusal ne sont pas les seules cellules cardiaques qui soient douées d'automatisme, mais ce sont les plus rapides, et elles imposent leur rythme au cœur. L'influx électrique né dans le nœud sinusal se propage dans l'oreillette droite, puis dans la paroi du septum interventriculaire, pour gagner par le faisceau de His les cellules contractiles du myocarde ventriculaire; la dépolarisation de l'oreillette se produit bien avant celle du ventricule et n'a que très peu d'effets mécaniques. La vitesse de conduction auriculoventriculaire (appréciable sur l'électrocardiogramme de surface par la durée de l'intervalle PR), déterminée par des résistances (électriques) variables entre cellules du tissu de conduction, est aussi sous la dépendance du SNA : une stimulation sympathique augmente cette vitesse, tandis qu'une stimulation parasympathique la diminue. C'est l'effet dit dromotrope du SNA. 


\subsubsection{La contractilité du myocarde}

Le couplage excitation-contraction dans la cellule ventriculaire est encore mal connu, mais le principal facteur de la puissance de la pompe cardiaque est la vitesse de raccourcissement des fibres contractiles. Là encore le SNA joue un rôle: le sympathique augmente la contractilité (c'est l'effet inotrope positif du sympathique); en revanche il y a peu, ou pas, de terminaisons nerveuses parasympathiques ventriculaires, et donc peu d'effet inotrope direct du parasympathique.

\subsubsection{La tension et la relaxation du myocarde}

De même, le sympathique augmente la tension au repos et la relaxation (= la distensibilité, i.e. la longueur de la fibre au repos) de la fibre myocardique, et augmente par là la puissance de la pompe. Ces effets sont dits tonotrope (tension), et lusitrope (relaxation). Ils augmentent l'effet Starling (dit aussi "loi fondamentale du cœur"), indépendant par ailleurs du SNA, qui permet au ventricule de se contracter d'autant plus efficacement qu'il est plus rempli, l'élongation de la fibre myocardique au repos étant plus important.

\subsubsection{Les résistances périphériques}

Elles sont sous l'influence du sympathique seul, principalement par l'intermédiaire de récepteurs noradrénergiques de type $\alpha$ (à la différence des récepteurs cardiaques, qui sont surtout de type $\beta$ [2]). L'effet du sympathique sur les résistances vasculaires est une vasoconstriction qui se traduit par une augmentation des résistances. Cet effet est sans doute celui qui est mis en place le plus rapidement dans le passage à l'orthostatisme (= la position debout), permettant de maintenir une pression artérielle efficace avant même que l'effet de l'augmentation du débit cardiaque ait pu se faire sentir.

\subsection{Mise en jeu physiologique et physiopathologique du SNA}

L'analyse spectrale du rythme cardiaque (identifié à la suite des intervalles de temps entre battements) et de la pression artérielle systolique, chez l'homme comme chez l'animal, met en évidence deux pics principaux : un pic, autour de $0.25 \mathrm{~Hz}$, correspond à la fréquence respiratoire, et est particulièrement net chez le sujet couché, en particulier en sommeil profond, et un autre, autour de $0.1 \mathrm{~Hz}$, représentatif des "ondes de Mayer", est un rythme endogène (on ne lui a pas trouvé d'origine physiologique extérieure au SCV) de basse fréquence bien visible en particulier sur un enregistrement effectué après passage à l'orthostatisme. Ces pics disparaissent en cas de blocage pharmacologique (électif : injection de $\beta$-bloquants pour la voie sympathique, d'atropine pour la voie parasympathique) des récepteurs du SNA. Ces modulations visibles sur un enregistrement de rythme cardiaque sont actuellement en clinique le principal moyen non invasif d'accès au SNA.

\subsubsection{L'arythmie sinusale respiratoire}

Elle n'a rien de pathologique, et est au contraire un élément du bon fonctionnement du SNA. Elle est définie par une augmentation de la fréquence cardiaque à l'inspiration, et une diminution à l'expiration. Il existe à ce mécanisme essentiellement deux origines, centrale et mécanique.

L'explication centrale est une interaction directe entre les centres autonomes qui contrôlent le cœur et le centre respiratoire bulbaire : le noyau dorsal du vague (alias cardiopneumoentérique). 
L'explication mécanique est fortement liée à la modification de la pression veineuse de retour due à l'inspiration: l'abaissement de la coupole diaphragmatique induit un pompage de sang abdominal dans la cage thoracique, avec distension de mécanorécepteurs (barorécepteurs du circuit veineux) situés dans l'oreillette droite, qui transmettent par la voie du nerf vague un message aux centres autonomes, provoquant une inhibition parasympathique, ce qui revient à une stimulation sympathique immédiate (réflexe de Bainbridge). Il faut y ajouter une stimulation parallèle de mécanorécepteurs pulmonaires à l'inspiration, qui produit les mêmes effets.

Enfin une explication mécanique locale directe, par étirement des fibres du nœud sinusal à l'inspiration (donc indépendamment du SNA) existe aussi, mais son importance est sans doute négligeable.

\subsubsection{Le passage à l'orthostatisme}

Au cours du passage à l'orthostatisme, il y a chute brutale de sang veineux vers les membres inférieurs, diminution de la pression veineuse de retour, diminution du débit cardiaque et donc de la pression artérielle au niveau des barorécepteurs artériels. Cette stimulation du baroréflexe artériel entraîne une élévation de la fréquence cardiaque, sans effets immédiats néanmoins, car la chute du retour veineux ne permet pas au débit cardiaque d'augmenter assez rapidement. C'est pourquoi la compensation de la pression artérielle lors du passage à l'orthostatisme se fait essentiellement par l'intermédiaire de la vasoconstriction périphérique, par une inhibition parasympathique consécutive à la chute de pression au niveau des barorécepteurs.

\subsubsection{L'exercice physique}

Les mêmes phénomènes que lors du passage à l'orthostatisme existent; il s'y ajoute aussi l'intervention d'un chémoréflexe musculaire (sensible aux pressions partielles en oxygène et en gaz carbonique, et un déplacement de la courbe stimulus-réponse du baroréflexe (réajustement d'une grandeur de consigne sur la pression artérielle) [15].

Mais chez les sujets sportifs entraînés, le tonus vagal est tel qu'il n'y a que très peu de réponse du sympathique à une chute de pression; au contraire les cas d'hypotension orthostatique à l'arrêt trop brusque de l'effort sont fréquents.

\subsubsection{L'insuffisance cardiaque}

Dans l'insuffisance cardiaque, en revanche, le débit cardiaque étant insuffisant, les résistances périphériques sont stimulées par la voie sympathique au maximum de leurs capacités, avec (régulation à moyen terme, hormonale) des taux très élevés de catécholamines circulantes.

\subsection{Commande pharmacologique du SNA}

Les médicaments du SNA ont une action soit centrale, soit périphérique. Ils stimulent ou bloquent les récepteurs, $\alpha$ ou $\beta$, à la noradrénaline, ainsi que les récepteurs, nicotiniques ou muscariniques (les récepteurs cardiaques sont muscariniques) à l'acétylcholine. Le plus connu des parasympatholytiques est l'atropine. On les utilise, expérimentalement ou en clinique, pour bloquer électivement une branche ou l'autre du SNA. Il existe aussi des $\alpha$ - et $\beta$-mimétiques, et des parasympathomimétiques. 


\section{Principes de la modélisation du SCV sous le contrôle du SNA}

\subsection{Problèmes généraux}

La construction d'un modèle du SCV sous la dépendance du SNA se heurte à des contraintes très fortes. Les variables mesurables (autant que possible de manière non invasive, c'est-à-dire par des instruments de mesure externes) sont bien peu nombreuses : pression artérielle en continu par brassard avec contre-pression (Finapres ${ }^{\mathrm{TM}}$ ), électrocardiogramme, respiration, électroencéphalogramme dans l'étude du sommeil. La pression veineuse centrale est par définition invasive, mais il peut être nécessaire d'en disposer sur des enregistrements animaux, par exemple, pour estimer le retour veineux. Quelles variables d'état considérer? Une variable d'"état sympathique" et une autre, d'"état parasympathique" sont de bons candidats pour décrire l'état interne du contrôleur.

Les conflits entre description microscopique (la cellule) et macroscopique (l'organisme) dans une modélisation à base physiologique sont représentatifs de l'opposition entre ce qu'on sait de la physiologie, souvent dans des détails très fins au niveau cellulaire, et ce qu'on peut en observer au niveau intégré du corps entier.

En dehors de l'observabilité, des problèmes d'identifiabilité sont à prévoir, et la construction d'un modèle doit en tenir compte, si on veut pouvoir s'en servir en clinique; mais suivant le problème physiopathologique considéré, tel ou tel aspect du mécanisme de commande peut être privilégié, aux dépens des autres, afin de rendre un modèle identifiable.

Enfin, signalons que l'intérêt des médecins et des physiologistes, dans notre pays, pour la modélisation mathématique est très variable; si certains y sont réfractaires, d'autres sont prêts à monter des expérimentations animales ou humaines pour tester une hypothèse.

\subsection{Cahier des charges physiologiques et applications à la modélisation}

\subsubsection{Pompe cardiaque}

La "loi fondamentale du cœur" (l'effet Starling) est que toute augmentation de la précharge (volume, ou pression au repos, avant la contraction) augmente la force de contraction du ventricule, non par augmentation de la vitesse de contraction (contractilité), mais par accroissement de la longueur de la fibre myocardique au repos. Ce mécanisme a pour effet d'égaliser les débits droit et gauche et d'éviter toute stase, et tout pompage inutile, dans la circulation pulmonaire. En revanche, toute augmentation de la postcharge diminue la contractilité du myocarde.

Un effet positif direct d'une augmentation de la fréquence cardiaque sur la contractilité, dit effet (de l'escalier de) Bowditch, est aussi à prendre en compte. Il est totalement indépendant et de l'effet Starling et du SNA, et est lié au largage intracellulaire de calcium: plus le cœur bat vite, et plus la fraction de temps pendant laquelle les canaux calciques membranaires sont ouverts est élevée; or le calcium intracellulaire est un déterminant essentiel de la contractilité musculaire.

L'intervention du SNA doit être modélisée à tous les niveaux du cœur. Sur le pacemaker (effet chronotrope), ce peut être par un oscillateur de type Hodgkin-Huxley à paramètres dépendant du SNA $[18,5]$, ou plus simplement par un oscillateur harmonique de fréquence dépendant directement du SNA [21]. Les effets bathmotrope (excitabilité générale du myocarde) et dromotrope (sorte d' "avance à l'allumage") peuvent être négligés dans un premier temps. L'effet inotrope est aussi essentiel à prendre en compte, la puissance de la pompe dépendant fortement d'une stimulation sympathique. 


\subsubsection{Circulation artérielle systémique}

L'impédance et la compliance des grosses artères doivent aussi être prises en compte dans la modélisation de la pression artérielle systémique, si on veut la mettre en rapport de manière réaliste avec le débit cardiaque et la résistance systémique.

\subsubsection{Baroréflexe}

La modélisation des non-linéarités est un aspect crucial. La voie sympathique est vraisemblablement une voie à retard par rapport à la voie parasympathique, et ce seul retard autorise l'apparition du rythme endogène à basse fréquence ; mais il faut noter que ce n'est pas la seule manière d'obtenir ce résultat. En particulier, modéliser le baroréflexe central comme un relais avec hystérésis (le déclenchement, l' "onset", du réflexe se produisant pour un point de consigne en pression plus élevé que son arrêt, l' "offset") permet, ainsi que le montre une analyse par la fonction de description, d'obtenir une stabilisation de la pression au prix de l'apparition d'un cycle limite (le " $0.1 \mathrm{~Hz}$ ") sans qu'il soit nécessaire d'introduire un retard. Ce rythme endogène de basse fréquence est-il transitoire ou non? On l'a décrit après passage à l'orthostatisme, et aussi dans certaines situations qui peuvent être liées à des perturbations passagères du baroréflexe. Si un tel phénomène hystérétique est avéré (il ne semble en particulier pas exister chez le chien au repos [11], et de même le $0.1 \mathrm{~Hz}$ est souvent totalement absent chez l'homme en position couchée au repos), sa quantification (par exemple par la largeur de la fenêtre d'hystérésis) peut être un élément précieux pour évaluer la sensibilité du baroréflexe.

\subsubsection{Résistances périphériques}

Elles doivent être décrites en incluant et l'autorégulation (qui, isolée, est dangereuse, puisqu'elle produit une ouverture des shunts périphériques en cas de diminution de la pression artérielle), et leur contrôle par le SNA (effets $\alpha$-adrénergiques).

\subsubsection{Circulation veineuse systémique}

La modélisation doit être celle d'une circulation à très basse pression, aidée par a/ une succion par la pompe cardiaque ("vis a fronte") ; b/ une pression résiduelle ayant passé la barrière artérioveineuse ("vis a tergo"); c/ une pression régionale (muscles) relayée par des valvules veineuses ("vis a latere"). Les interactions mécaniques régionales avec les mouvements respiratoires (veine cave supérieure et oreillette droite) doivent prendre en compte la très grande compliance des veines, et leur fonction fondamentale de réservoir de sang.

\subsubsection{Petite circulation et mouvements respiratoires}

La petite circulation représente moins de $10 \%$ du volume sanguin circulant; la réserve de sang pulmonaire est d'environ 6 systoles ( 6 fois le volume d'éjection moyen), et cette marge permet au SCV de s'adapter dans l'urgence à un effondrement de la pression veineuse de retour.

$\mathrm{Si}$ on prend en compte les concentrations artérielle et veineuse en oxygène, leur dépendance de la fréquence et de l'amplitude des mouvements respiratoires est à intégrer au modèle ; mais il faut surtout rendre compte de l'arythmie sinusale respiratoire, du moins dans sa partie mécanique en rendant la pression veineuse centrale dépendante de ces mouvements. 


\subsection{7 États physiopathologiques complexes}

Il s'agit de l'exercice physique extrême (chémoréflexes: $\left[\mathrm{O}_{2}\right],\left[\mathrm{CO}_{2}\right], \mathrm{pH}$ ), de pathologies impliquant des perturbations importantes du SNA (p.ex. diabète avec neuropathie), et du sommeil paradoxal. Le sommeil paradoxal est un état physiologique du système nerveux central au cours duquel des interactions très complexes peuvent survenir entre notamment la réticulée et les centres cardiorespiratoires du bulbe, avec déconnection concomitante du contrôle par l'hypothalamus et le cortex, mais surtout une interaction moindre que dans le sommeil profond entre centre respiratoire et noyau ambigu (donc une diminution de l'arythmie sinusale respiratoire) [1]. C'est pourquoi une identification des paramètres du SNA doit prendre en compte les états de vigilance et de comportement.

\section{3 État de l'art}

Une description de base du SCV peut être trouvée dans [16]. Un point de vue plus général sur la physiologie intégrative peut être trouvé dans [4], et le petit atlas de Silbernagl et Despopoulos [19] est une référence précieuse pour la description des grandes régulations physiologiques. Toujours du côté de la physiologie, la revue de Melchior [13] traite de la réponse à l'orthostatisme. Eckberg [7] a mené de nombreuses expériences décrivant le fonctionnement du baroréflexe; Taher [20] traite en particulier du problème de l'hystérésis dans le baroréflexe. Le livre de Levy et Schwartz [12] fait un point récent complet sur le contrôle vagal, et aussi accessoirement sur le contrôle sympathique. Schwaber [17] étudie le fonctionnement des mécanismes centraux du baroréflexe (au niveau du NTS).

Les interactions entre mathématiciens, automaticiens, et physiologistes ne datent pas d'hier. En particulier, Guyton [8] a développé dès les années 60 un modèle analogique complet du SCV, incluant aussi le rein (ce qui est nécessaire pour étudier l'hypertension artérielle); de même, Milhorn [14] propose des principes de modélisation par schémas-blocs du SCV, et pas seulement par des outils linéaires. Kitney [10] a dès 1979 proposé l'analyse du SCV par la fonction de description. Plus récemment, à l'aide de modèles à base d'équations différentielles, Vermeiren [21], par la méthode de la fonction de description, Seidel [18], et Cavalcanti [3], par une analyse de continuation-bifurcation suivant un paramètre "retard sur la voie du baroréflexe", ont rendu compte du rythme endogène de basse fréquence. Le débit cardiaque et la pression artérielle, avec la résistance systémique, sont étudiés dans [22]. Un modèle du SCV récent et complet, mais non pulsatile, est celui de Kappel [9].

\subsection{Diverses pathologies et disciplines médicales concernées}

En cardiologie, il a été montré que sur l'ensemble d'une population, un mauvais état du SNA augmentait les risques de mort subite chez les survivants après infarctus du myocarde.

En neurologie, on étudie des pathologies à l'origine d'une atteinte du SNA (p. ex. neuropathie diabétique) ou liées à un dysfonctionnement du SNA (syndrome des apnées du sommeil d'origine centrale, syndrome des myoclonies nocturnes, notamment).

En pédiatrie, un défaut de maturation du SNA a été proposé comme facteur de risque de mort subite inexpliquée du nourrisson.

En obstétrique, il est connu de longue date qu'une chute de la fréquence cardiaque fotale suivie d'un plateau (perte de variabilité) est un signe de souffrance fotale aiguë.

Ces disciplines, ainsi que d'autres (anesthésie-réanimation pour surveillance-diagnostic au bloc opératoire ou en unité de soins intensifs) pourraient bénéficier d'un modèle du SCV et de son contrôle 
par le SNA qui leur donnerait accès aux paramètres internes du SCV.

\section{Références}

[1] Benoit, O., Foret, J. Le sommeil humain, bases expérimentales et physiopathologiques. Masson, Paris, 1992.

[2] Bioulac, B. La neurotransmission dans le système nerveux végétatif. La revue du praticien, 31 (37): 2605-2631, 1981

[3] Cavalcanti, S., Belardinelli, E. Modeling of Cardiovascular Variability Using a Differential Equation. IEEE-BME, 34 (10): 982-988, 1996.

[4] Chauvet, G. Traité de physiologie théorique (3 tomes). Masson, Paris, 1987.

[5] Clairambault, J. A model of the autonomic control of heart rate at the pacemaker cell level through G-proteins. Actes de la $17^{\mathrm{e}}$ conférence internationale IEEE-EMBS, 1379-1380, Montréal, septembre 1995.

[6] Clairambault, J., Claude, D. Systèmes dynamiques et biologie : quelques exemples. Séminaire T. I. P. E. sur les systèmes dynamiques. Journée de formation (ENSTA-UPS) pour les professeurs de Mathématiques Spéciales (organisateur: Max Bezard). ENSTA, Paris, mai 1996.

[7] Eckberg, D.L., Rea, R.F., Andersson, O.K., Hedner, T., Prnow, J., Lundberg, J.M., Wallin, B.G. Baroreflex modulation of sympathetic activity and sympathetic neurotransmitters in humans. Acta Physiol. Scand., 133: 221-231, 1988.

[8] Guyton. A.C. Arterial Pressure and Hypertension. Saunders, Philadelphie, 1980.

[9] Kappel, F., Peer, R.O. A mathematical model for fundamental regulation processes in the cardiovascular system. J. Math. Biol., 31: 611-631, 1993.

[10] Kitney, R.I. A nonlinear model for studying oscillations in the blood pressure control system. $J$. Biomed. Eng., 1 (2): 89-98, 1979.

[11] Levison, W.H., Barnett, G.O., Jackson, W.D. Nonlinear analysis of the baroreceptor reflex system. Circ. Res., 18: 673-682, 1966.

[12] Levy, M.N., Scwartz, P.J., Eds. Vagal control of the heart. Futura, New-York, 1994

[13] Melchior, F.M., Sirnivasan, R.S., Charles, J.B. Mathematical modeling of human cardiovascular system for simulation of orthostatic response. Am. J. Physiol., 262: H1920-H1933, 1992.

[14] Milhorn, H.T. The application of control theory to physiological systems. Saunders, Philadelphie, 1966.

[15] Papelier, Y. Adaptation cardiovasculaire à l'exercice physique chez le sportif : rôle de l'interaction entre baroréflexes et chémoréflexe musculaire. Thèse de doctorat en sciences et techniques des activités physiques et sportives, Université Paris Sud, juin 1994.

[16] Hoppensteadt, F.C., Peskin, C.S. Mathematics in Medicine and the Life Sciences. Springer Texts in Applied Mathematics, 1992.

[17] Schwaber, J.S., Graves, E.B., Paton, J.F.R. Computational modeling of neuronal dynamics for systems analysis: application to neurons of the cardiorespiratory NTS in the rat. Brain Res., 604: 126-141, 1993.

[18] Seidel, H., Herzel, H. Modelling Heart Rate Variability Due to Respiration and Baroreflex. In : E. Mosekilde, O.G. Mouritsen, Eds. Modelling the Dynamics of Biological Systems. Springer Series in Synergetics, 1995. 
[19] SilBernagl, S., Despopoulos, A. Atlas de poche de physiologie. Médecine-Sciences, Flammarion, Paris, $\left(4^{\mathrm{e}}\right.$ de la $2^{\mathrm{e}}$ édition française) 1995.

[20] Taher, M., Cecchini, A.B.P., Allen, M.A., Gobran, S.R., Gorman, R.C., Guthrie, B.L., Lingenfelter, K.A., Rabbany, S.Y., Rolchigo, P.M., Melbin, J., Noordergraaf, A. Baroreceptor responses derived from a fundamental concept. Ann. Biomed. Eng., 16: 429-443, 1988.

[21] Vermeiren, C. Analyse et modélisation du système cardio-vasculaire et sa régulation à court terme par le système nerveux autonome. Thèse de doctorat en génie biologique et médical, Université Paris Val de Marne, décembre 1996.

[22] Wesseling, K.H., Jansen, J.R.C., Settels, J.J., Schreuder, J.J. The computation of aortic flow from pressure in humans using a nonlinear, three-element model. J. Appl. Physiol., 74 (5): 2566-2573, 1993. 